\title{
EXPERIMENTAL INVESTIGATION OF GAS/SHEAR THINNING FLUID FLOW IN A HORIZONTAL PIPE
}

\author{
Serag Moustafa M. Alfareq ${ }^{1}$, Abinash Barooah ${ }^{2}$, Mohammad Azizur Rahman ${ }^{2}$, Sohrab ${ }^{4}$ Zendehboudi $^{1}$ \\ ${ }^{1}$ Memorial University of Newfoundland, St. John's, NL, Canada \\ ${ }^{2}$ Texas A\&M University at Qatar, Doha, Qatar
}

\begin{abstract}
The objective of the study focuses on gas/non-Newtonian liquid horizontal flow in a pipeline. The gas and the liquid flow rates were varied over a wide range of flow conditions. The experiment was conducted to determine the pressure drop using a transparent pipe of $76.2 \mathrm{~mm}$ diameter and $2.44 \mathrm{~m}$ long horizontal pipe. Xanthan gum solution was used to simulate non-Newtonian fluid. The pressure drop gradient model of Lockhart-Martinelli was modified for gas/shear thinning fluid flow. The theoretical predictions of pressure loss were compared with the experimental data for air/shear thinning fluid flow results. From the experimental work, it was observed that the pressure drop was directly proportional to the concentration of Xanthan gum (shear thinning fluid). The flow regimes of bubbly, slug, plug and wavy flow were also captured by a high-speed camera.
\end{abstract}

KEY WORDS: Gas/non-Newtonian liquid; shear thinning fluid; Pressure drop; Two-phase flow rates; visualization.

\section{INTRODUCTION}

Multiphase units are used in a wide range of commercial industries, such as gas and oil, refining, and mining in regards to some broad fields of matter [1]. The term multiphase flow refers to fluid flow that encompasses more than one component or a phase or component flowing throughout a pipeline [2]. There is a greater variety of applications of multiphase flow than single-phase flow because of differences in density. Some important applications are sizing of process equipment, liquid management, and well drilling operations. Accordingly, it is highly challenging, yet critical, for engineers to select the right multi-phase pressure drop in liquid accumulation and pigging [3]. To prevent problems such as large liquid surges, flow instabilities, and high liquid hold up, pipe diameter and pump design selection needs precise pressure drop measurements. Body force (gravitation), acceleration, and friction loss impact the pressure loss in a tube flowing upward in an inclined angle. The losses are mutually dependent and non-linear. To illustrate, the gravitation force leads to the reduction of pressure. Accordingly, the density changes and as a result, acceleration takes part. Nevertheless, it is possible to neglect this dependency for small distances. The acceleration pressure losses throughout pipelines and wellbores in most cases are very little. Additionally, for horizontal flow, there is no gravitational pressure loss. Therefore, these losses are ignored during the pressure drop calculations. As a result, the total pressure loss in pipeline or wellbore can be explained as the frictional pressure loss in the pipeline.

Several correlations are employed to precisely calculate the pressure drop in a single-phase flow in a wellbore or pipeline or wellbore, such as Weymouth Correlation, Panhandle Correlation, Blasius Correlation, Darcy-Weisbach Correlation, Fanning Correlation. Typically, flows in wellbores and pipelines in the field and industry are turbulent. This study used the Fanning correlation for calculating the pressure loss for both

marahman@tamu.edu 
single-phase and two-phase flows in a horizontal orientation. However, Single phase pressure loss calculations and multiphase pressure loss calculations are not the same. Hence, correlations must be modified to be applied to multiphase cases. To adequately predict the pressure loss in a two-phase (liquid and air) flow, the fundamental fluid parameters that require the modification are the viscosity of the twophase mixture, density of the two-phase mixture, velocity of the two-phase mixture. As mixture properties need to be used, it is required to determine the gas and liquid in-situ volume fractions through the pipe (Welling an Associates, 1999). The frictional and hydrostatic pressure losses are modified to compute the pressure loss in a multiphase flow in correlations. The correlations are Duns \& Ros Correlation, Hasan \& Kabir Correlation, Ghajar \& Bhaguat Correlation, Dukler et al. Correlation, Beggs and Brill Correlation, Lockhart-Martinelli (L-M) Correlation

The first research on multiphase flow was conducted by Lockhart- Martinelli in 1949. His method had been standard in that period and achieved satisfactory results regarding two-phase flow in a horizontal pipe. Nevertheless, there are inconsistencies when applying his method to flows with three and more phases. Hence, in 1954, Baker carried out experiments that resulted in important changes in the equations of Lockhart- Martinelli regarding flow patterns in horizontal pipelines. More precisely, Baker (1954) proposed new correlations for all flow regimes for two-phase flow. Nonetheless, Dukler et al. (1964) did an experiment with Lockhart-Martinelli's and Baker's pressure drop correlations applied to a large number of data points. Their experiment revealed that correlations of Lockhart-Martinelli are superior in approximating flow regimes, apart from the case of wavy flow. So far, only several studies have focused the pressure drop features related to flow pattern [4]. New knowledge about multiphase flow regime development will significantly contribute to financial savings in the industrial sector. It has been established that the LockhartMartinelli correlation is appropriate for two-phase flows at low and moderate pressures. However, it is recommended to use the modified models of Martinelli and Nelson (1948) and Thom (1964) if applied at higher pressures [5]. In this paper three correlations i.e. Lockhart-Martinelli, Dziubinski - Chhabra, Ruiz Viera et al. correlations are compared with the experimental data set to check with model can predict the pressure drop more accurately [6,7].

Another important parameter in multiphase flow is the flow pattern of the two-phase flow. Typically, the term two-phase flow indicates the simultaneous flow of liquid and gas through a pipeline system. Furthermore, as the interface of liquid and gas is deformable, it is challenging to predict which regions are occupied by liquid and by gas phase. During the flow of two phases throughout a pipeline, various types of interface distribution can be formed. Typical distributions are:

- Annular flow - the liquid flow takes a form on a film and it is located on the wall of the pipe.

- Stratified flow - the gas is lighter than liquid and it flows on the top; two phases are separated.

- Slug flow - gas bubbles take a form of a large slug, commonly in the shape of a bullet.

- Bubbly flow - small-sized bubbles are dispersed with liquid.

Typically, the multiphase flow rate is characterized by its superficial flow velocity and liquid superficial velocity. The superficial velocity is the term used to describe the gas-liquid multiphase flow. It is also used in the flow regime maps. Under the condition of the superficial velocity, the fluid flows without the other fluid being present. Hence, there is no slip between the phases to consider [8]. Put differently, following Crowe [9], superficial velocity refers to the total amount of fluid throughput divided by the cross-section area of the pipe. The volume fraction is the ratio of the gas or liquid flow rate and the total fluid flow rate. The input volume fraction of individual phases can be different than in-situ volume fraction. Also, the buoyancy force and gravity significantly impact heat transfer in the pipeline system, volume fraction, pressure drop, and the flow regime [10]. The flow regimes for horizontal pipe are illustrated in Figure 1. 


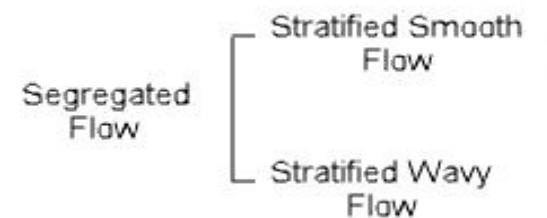

Flow
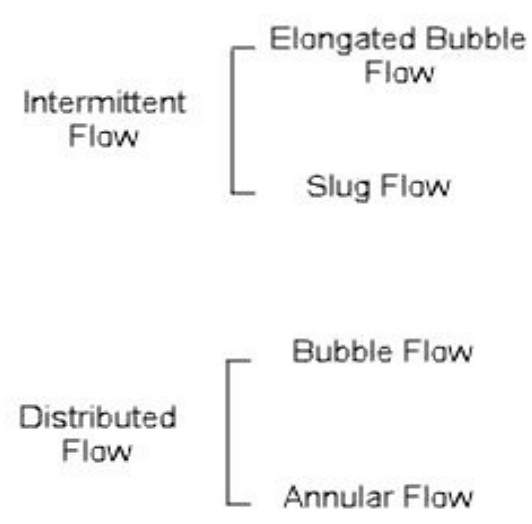
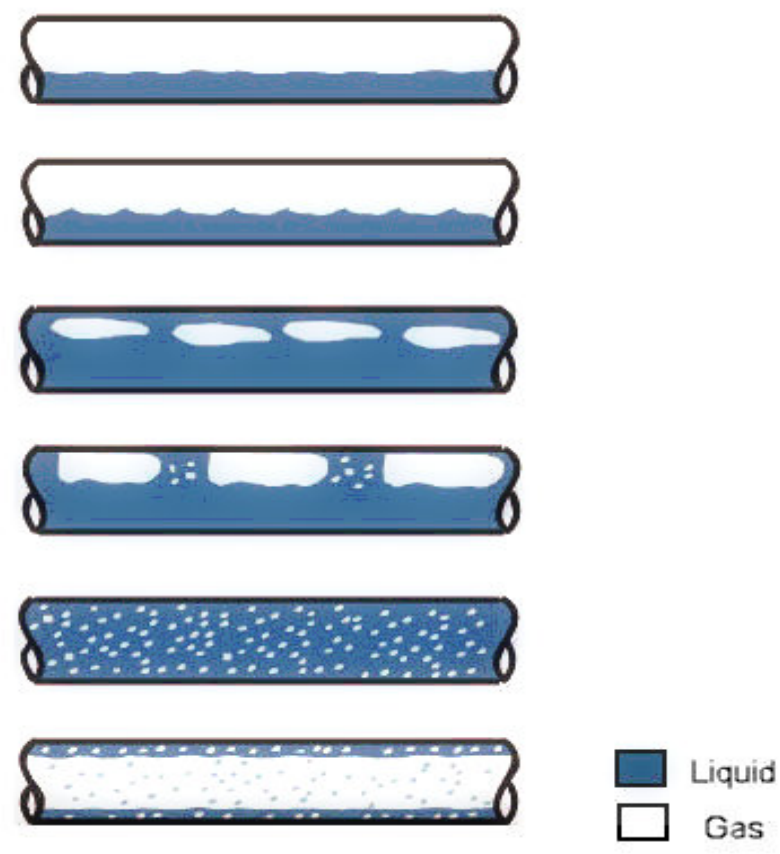

Figure 1 Gas-Liquid Flow Patterns in Horizontal Pipelines (Adapted from Hubbard, 1966 [11])

It is critical to comprehend the impact of the frictional elements in pressure loss to improve the accuracy of the process system design [12]. There is a wide use of two-phase flow in the process and related industries as it includes various flow regimes, such as annular, slug, stratified, and bubble. There are great pressure fluctuations in the slug and stratified regimes that can impair the equipment, as they impact mass and transfer phenomena of the equipment [13]. Although a significant amount of research has been carried out on multiphase flow, still a lot of uncertainty exists on the different parameters affecting the pressure drop. There is also a lack of proper validation and comparison of different correlations with experimental data. This study aims to accurately evaluating the pressure drop for non-Newtonian two-phase flow. Moreover, its aim is to predict the flow patterns for Newtonian and non-Newtonian fluid for different flow rates. Finally, it will analyze the effect of the changes in non-Newtonian fluid concentrations and the flow rates on the pressure drop. Additionally, the experimental result is also compared with different correlations to identify their accuracy.

\section{EXPERIMENTAL SETUP AND PROCEDURE}

The flow loop refers to a 65-meter pipe closed-loop system. A polyvinyl chloride pipe (PVC) is used to pump the liquid from the tank. To visualize the flow, there are installed transparent horizontal pipe sections. Different temperature and pressure sensors are used to measure the liquid and gas flow rates as illustrated in Figure 2 in more details. This flow loop is employed to produce the two-phase flow. It is done by mixing liquid from the liquid line and airflow from the air line. The air injection pipe comprises of two sizes - 1inch and 0.5-inch needed for various air flow volumes. Control valves are employed for compressing airflow and throttling the liquid. In this way, the flow regimes are created, and the flow conditions controlled. Data acquisition system measures process parameters. 


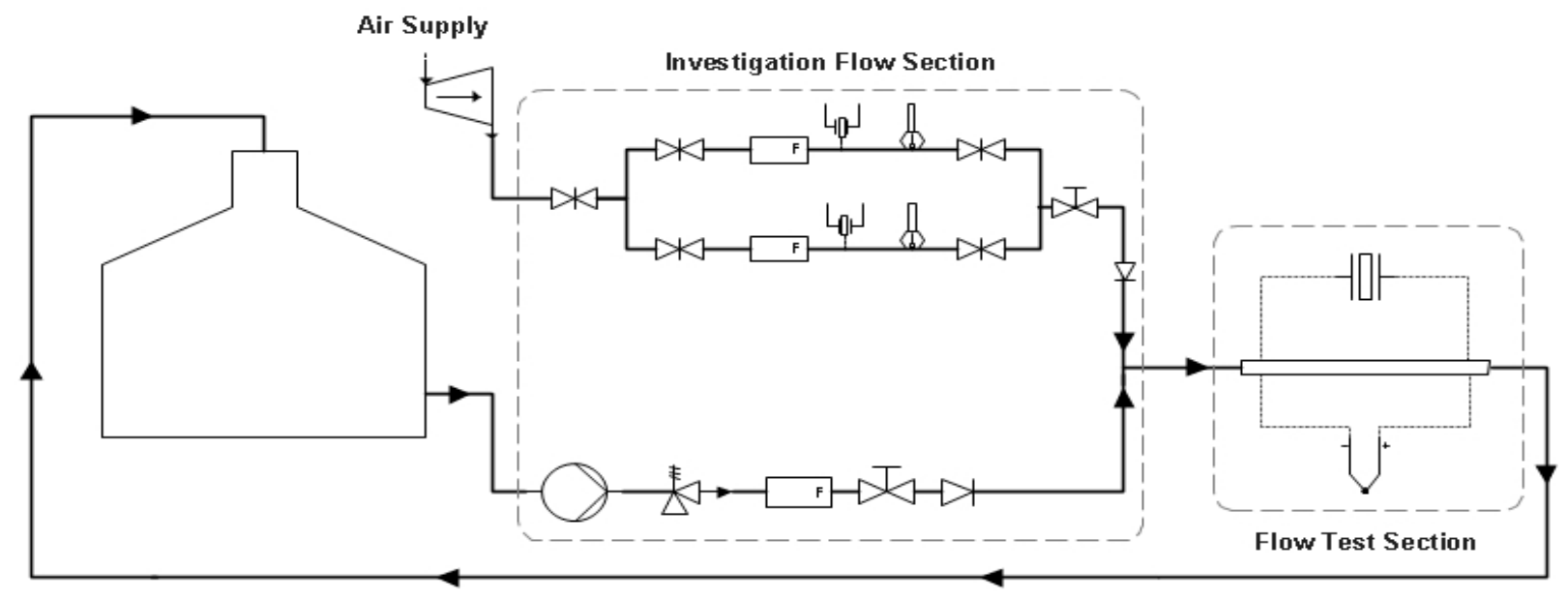

Figure 2. Schematic of The Flow Loop

There are four Omega PX603 series cable style pressure transducers in the flow loop, compatible with gas and liquid for measuring the pressure drop. In the air lines, two models of PX603-200G5V were used for 0 to 200 psi (0 to $1378.95 \mathrm{kPa})$. In the liquid line, the model PX603-100G5V was used for 0 to $100 \mathrm{psi}(0$ $689.48 \mathrm{kPa}$ ). Transducers are attached to the data acquisition system and they create a 1-5 V output signal. Each transducer has a 1/4-inch male National Pipe Thread Taper (NPT) fitting. It is required for the installation into the flow loop. To estimate the pressure gradients, the pressure should be measured when the test section begins and when it ends. Omega Differential Pressure Range, 0 To 5 V DC (direct current) Output, $2 \mathrm{~m}$ (6ft) Cable Termination [14] was employed in the experiment to estimate the differential pressure. It has a full scale of $5 \mathrm{psi}(34.473 \mathrm{kPa})$ and an accuracy of $+/-0.008$. With this accuracy, the error is around $28 \mathrm{~Pa}$. Comark C9555 pressure meter was employed to compare its measurements with those of the $\mathrm{dP}$ (differential pressure) cell sensor measurements. It is both very fast and accurate. Also, it includes twin inputs for gauge pressure measurement. In general, applications include calibration of other instruments, service and maintenance, process pressures, laboratories and clean rooms, air conditioning filters, and boiler flue draft [15].

There are two electro-pneumatic ball control valves for controlling the flow loop system. One is installed into the air lines, and the other in the liquid line. They must be installed promptly before joining the compressed air and liquid lines. A 1-inch valve is used to control the gas flow in the air line, whereas a 3inch valve is in charge of controlling the liquid flow in the PVC pipe. Both are connected to VRC VE700 electro-pneumatic positioners and Bettis pneumatic actuators. There is a pressure relief valve on the flow loop whose pressure is set to 100 psi on the basis of different flow-rates in gallon per minute (GPM) versus set pressure and seat size. The valve opens when the pressure is higher than $100 \mathrm{psi}(690 \mathrm{kPa})$, and then the liquid is let to get into the drain. Hence, the valve is configurated at $690 \mathrm{kPa}$. Accordingly, the working pressure rating of the pipe of $130 \mathrm{psi}(900 \mathrm{kPa})$, is also the maximum pressure in the pipe. The pressure set for other components is $150 \mathrm{psi}(1030 \mathrm{kPa})$. Additionally, a pressure regulator is present which represents a specialized control valve for reducing the upstream supply pressure level to a determined constant downstream pressure.

A DAQ (Data acquisition system) was used to collect input signals from the meters and sensors. What is more, it emits the signals to the control valves in the air line and liquid. When using the DAQ system, the user is able to decide and to control which amount of fluid will enter the system and circulate through it. The SignalExpress software monitor displays flow rates, pressure signals, and temperature signals of the system. A Mega Speed MS55K Digital Camera System is used to improve the visualization of the flow loop and helps to identify the different flow regimes. The High-Speed Imaging Software is a user-friendly image capture program, yet with a high capacity. It is used for rapid identification, diagnosis, and solving problems by taking videos. The transformation of images to the PC occurs in real-time. Moreover, a marsh funnel 
viscometer and rotary viscometer was used for measurement of viscosity and mud balance was used for measurement of density.

\section{RESULTS AND DISCUSSION}

\subsection{Pressure Drop Measurement}

Three operating pressures, namely 15 psi $(40 \mathrm{~Hz}), 20 \mathrm{psi}(50 \mathrm{~Hz})$, and $25 \mathrm{psi}(60 \mathrm{~Hz})$, were used to perform experiments. Operating the setup at $20 \mathrm{psi}(50 \mathrm{~Hz})$ is the optimum condition of the flow loop because the best results of flow regime tests and the pressure drop were obtained in this case.

3.1.1 Single-phase flow. Figures 3 and 4 show the pressure drop for non-Newtonian fluid (xanthan gum solution). It can be seen that pressure drop rises with rising operating pressure and the flow rates for singlephase flow. A satisfactory agreement with the experimental results was presented by the empirical models. The best approach in regard to the experimental results of used xanthan gum solution concentrations is the Herschel-Bulkley model. Also, more pressure drops with the $2 \mathrm{~g} / \mathrm{L}$. Therefore, at a higher viscosity and higher concentration, xanthan gum solutions can lead to an additional decrease in pressure in horizontal single-phase flow.

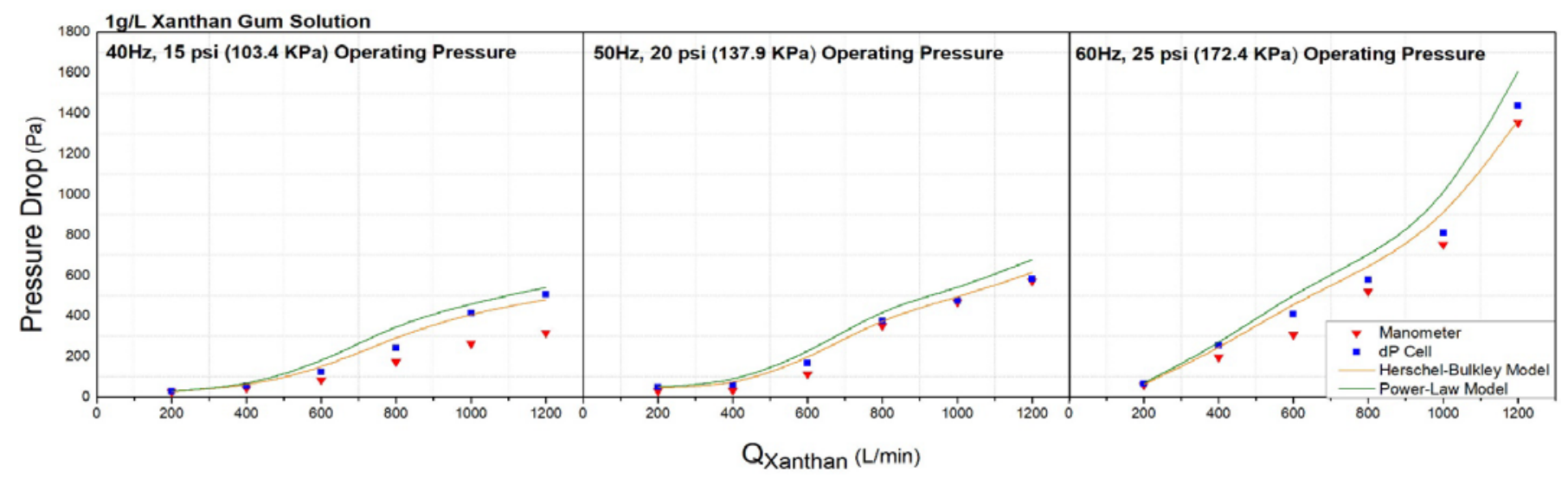

Figure 3 1g/L Xanthan Gum Solution - Experimental and Correlations Pressure Drop Results

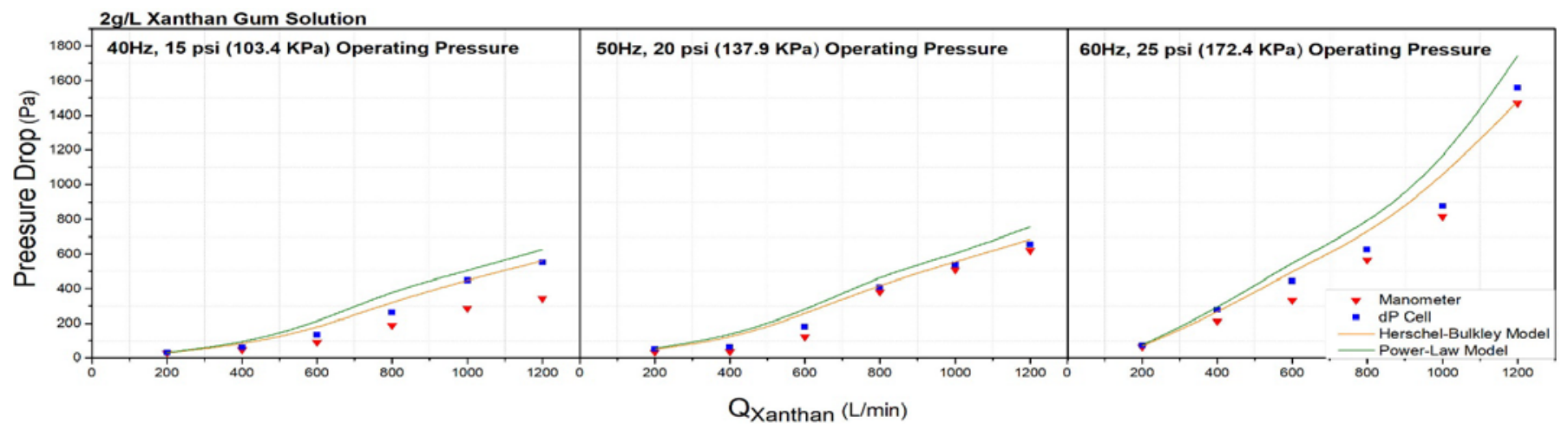

Figure 4 2g/L Xanthan Gum Solution - Experimental and Correlations Pressure Drop Results 
3.1.2 Two-phase flow. The review of Figures 5 and 6 shows the growth in the pressure drop along with the rise in the operating pressure and xanthan gum solution flow rates when airflow rates are constant in the horizontal non-Newtonian two-phase flow. Furthermore, in comparison to the $1 \mathrm{~g} / \mathrm{L}$ concentration case, the case of $2 \mathrm{~g} / \mathrm{L}$ concentration represents the most important development of the pressure drop. Moreover, it is observed that the highest values of the pressured drop are reached at the operating pressure of $25 \mathrm{psi}(60 \mathrm{~Hz})$. In addition, in comparison to the case of the $1 \mathrm{~g} / \mathrm{L}$ xanthan gum concentration, the $2 \mathrm{~g} / \mathrm{L}$ xanthan gum concentration flows cause the highest growth of pressure drop. Hence, xanthan gum solutions generate an additional pressure drop in horizontal non-Newtonian two-phase flow at a higher viscosity and higher concentration. According to the empirical correlations, there is satisfactory matching with the experimental results. The Lockhart-Martinelli model exhibits the most accurate match with the obtained empirical results.

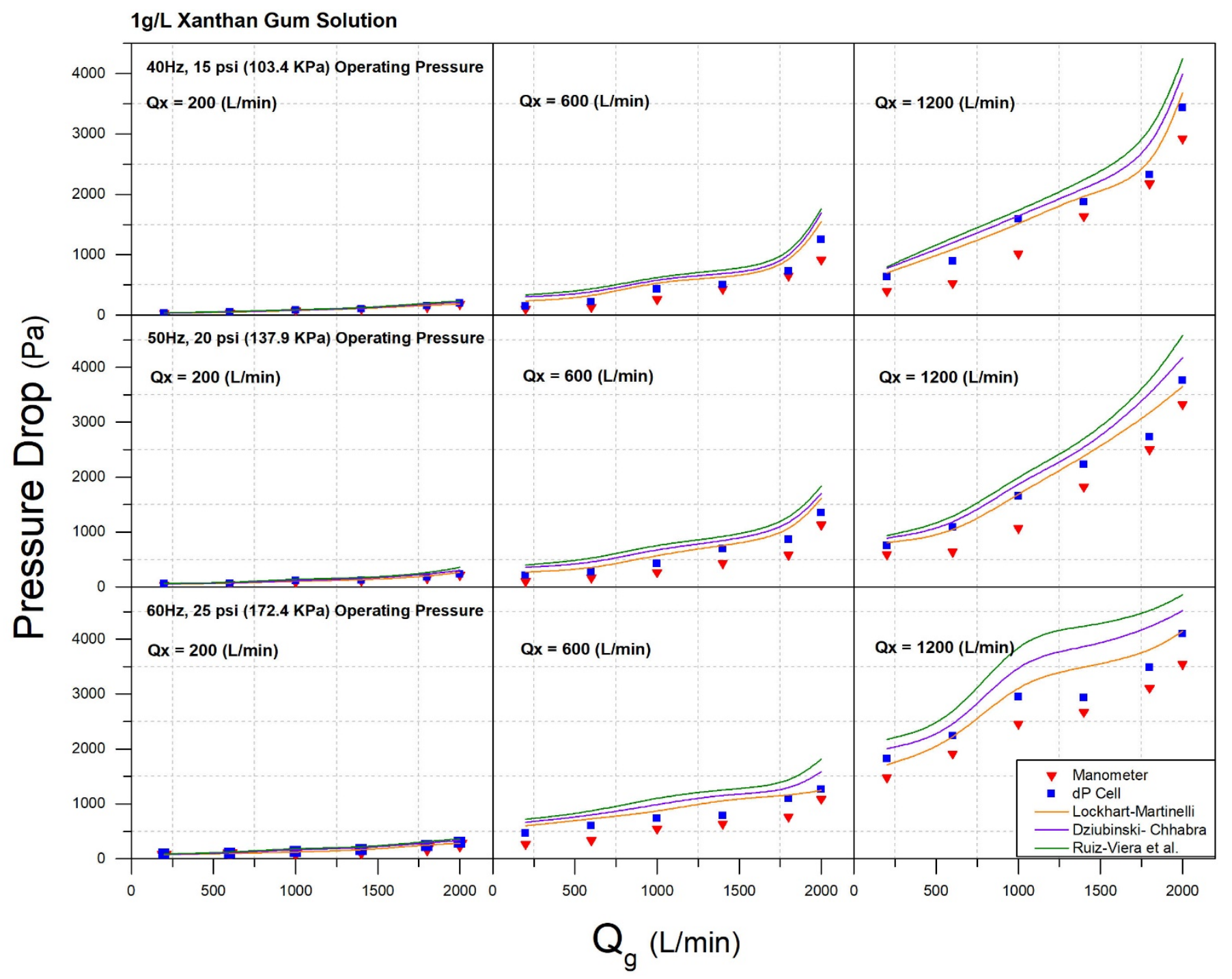

Figure 5 Two-Phase Air Flow and 1g/L Xanthan Gum Solution - Experimental and Correlations Pressure Drop Results 


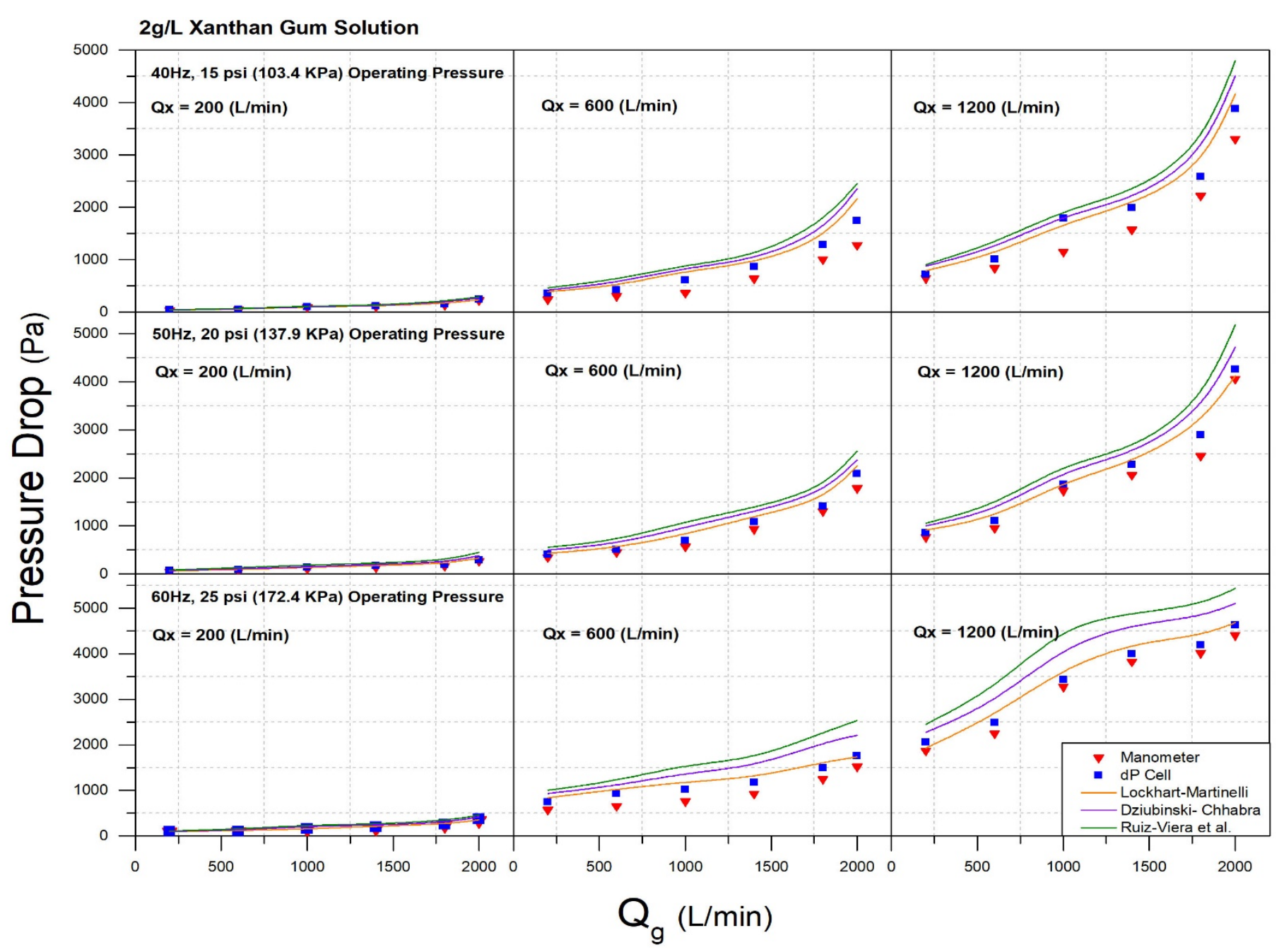

Figure 6 Two-Phase Air Flow and 2g/L Xanthan Gum Solution - Experimental and Correlations Pressure Drop Results

\subsection{Flow Regime Visualization}

The operating pressure of $20 \mathrm{psi}(50 \mathrm{~Hz})$ was used to perform the flow regimes experiments for Newtonian and $1 \mathrm{~g} / \mathrm{L}$-concentration non-Newtonian flow.

The flow regime images presented in Figure 7 taken by the high-speed camera, Mega Speed MS55K. correspond to three Newtonian flow regimes used in this study. The sequential visualization shows bubble, plug, and slug flow with the rise in the airflow rate. Figure 8 shows standard bubble, plug and slug flow regimes of $1 \mathrm{~g} / \mathrm{L}$-concentration non-Newtonian flow (xantham gum solution). They were taken with the highspeed camera. It was not possible to visualize the $2 \mathrm{~g} / \mathrm{L}$ xanthan gum solution flow because of a murky flow caused by the more considerable amount of xanthan gum powder.

Non-Newtonian flow required more amounts of air to produce bubble flow, plug flow, and slug flow regime comparing to Newtonian flow case. 


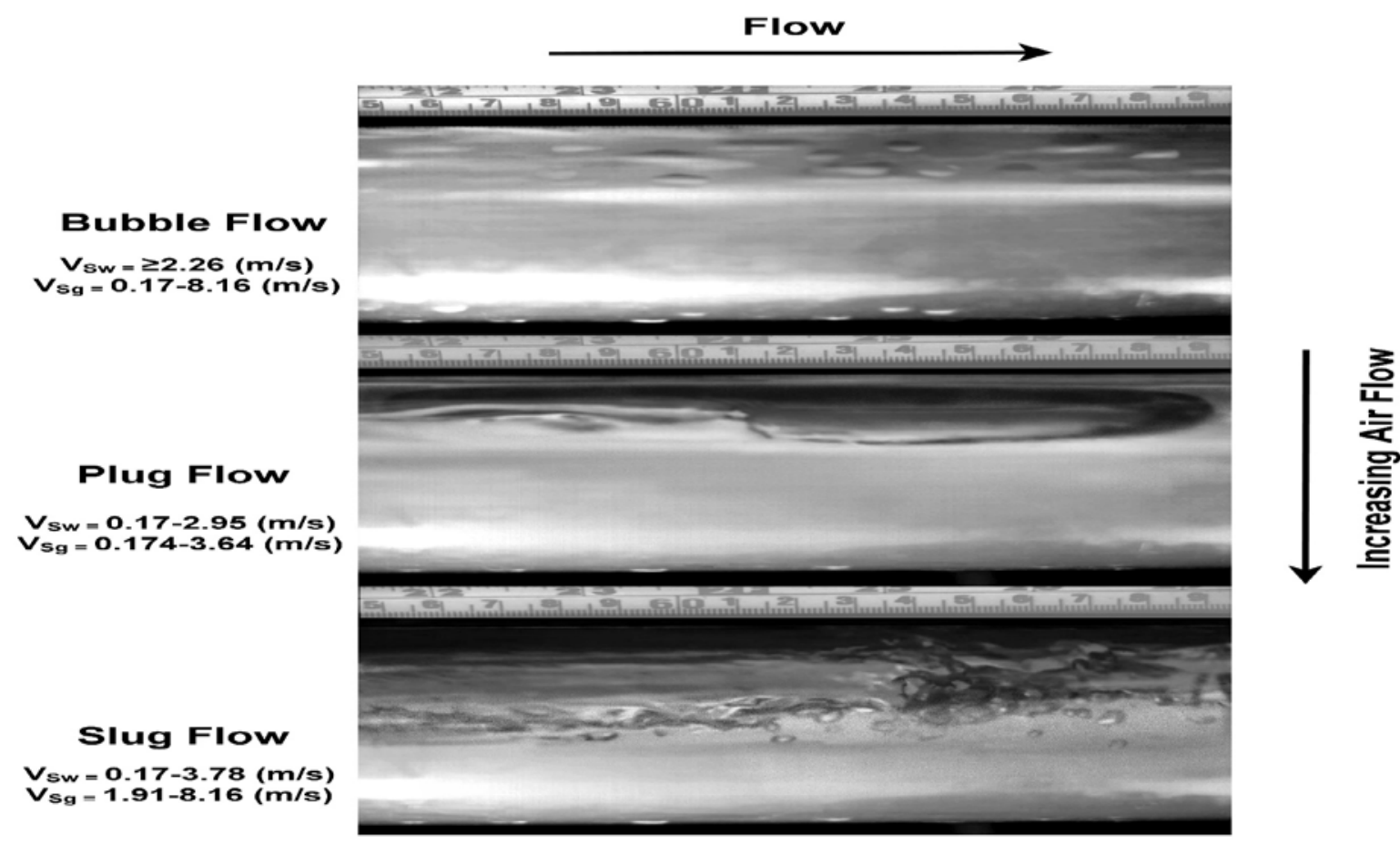

Figure 7 Newtonian Flow Regimes Visualized by High-Speed Camera Mega Speed MS55K

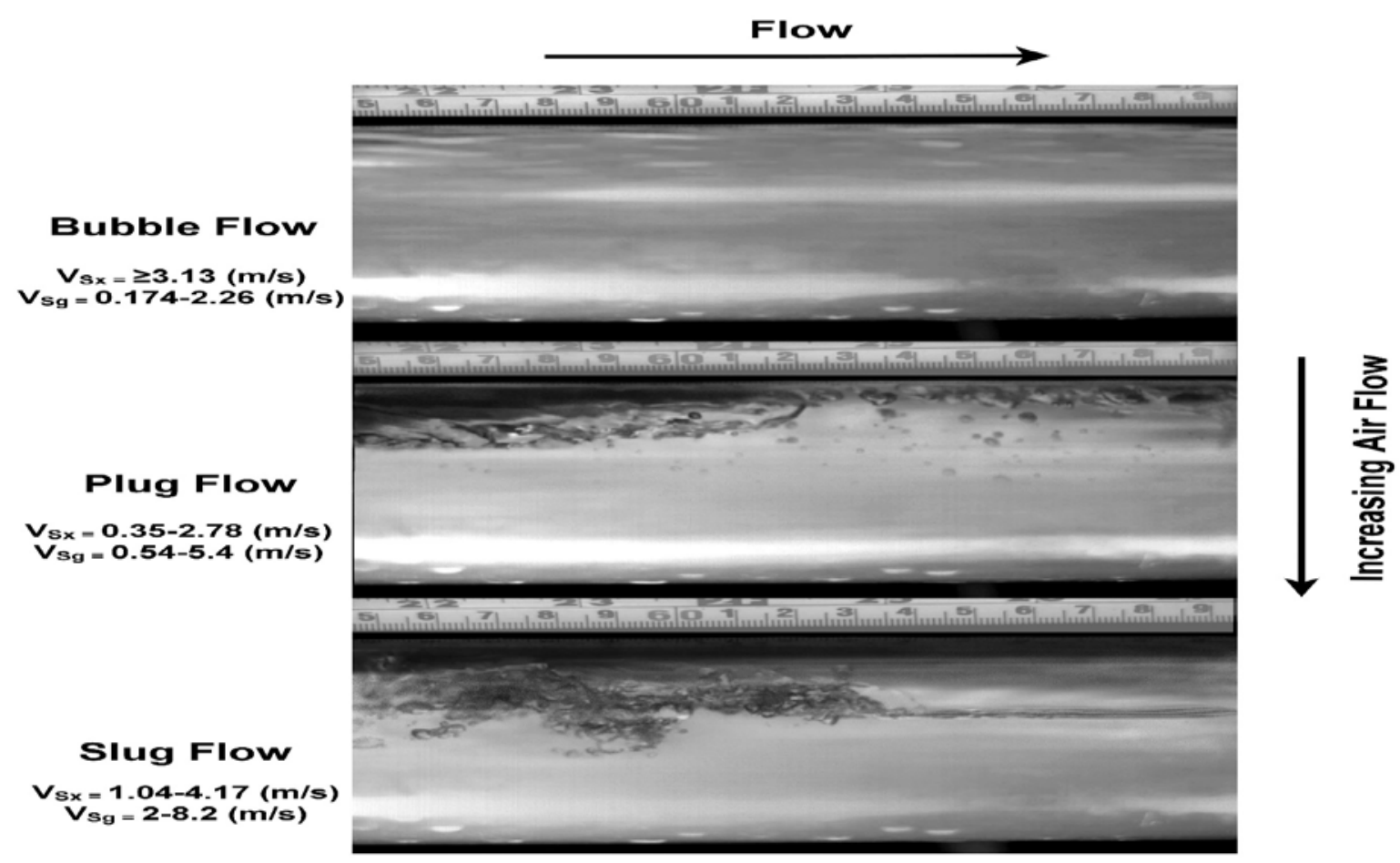

Figure 8 Non-Newtonian Flow Regimes Visualized by High-Speed Camera Mega Speed MS55K 


\section{CONCLUSIONS}

To detect the data trends, the pressure drop was plotted over the air and liquid flow rates. The comparison of the experimental data with different empirical correlations provided good results, thus showing the reliability of the correlations to predict the pressure drop for single-phase and two-phase (liquid-gas) flow. It was found that the single-phase pressure drop rises along with the rise in the flow rate for non-Newtonian flow test. It was noticed that the two-phase pressure drop rises with the rise in the air flow rate (air superficial velocity) for air-water two-phase flow. It happens because air phase disturbs the flow and creates turbulence hence, extra pressure loss happens in the mixture of air-water flow. Moreover, the experiment explained the impact of the rising xanthan gum solution concentrations and the flow rates on the pressure drop of xanthan gum solution/air flow. It was definitely observed that the non-Newtonian pressure drop increases with the rise in xanthan gum solution concentration. A higher viscosity liquid establishes extra frictional shear forces along the pipe leading to a more substantial pressure drop. Moreover, it was observed that Lockhart-Martinelli model exhibits the best match with two-phase experimental results due to the presumption of considering each phase flow individually and an accurate representation of various parameters.

The study accomplished flow regime visualizations for Newtonian and non-Newtonian flow. Only Slug flow, plug flow, and bubble flow were observed for both the fluids. The flow loop setup is not adequate in providing annular, wavy, or stratified flow regimes. It was influenced by the flow rates of the fluids, their physical properties and the setup design (pipe diameter). In order for the flow loop to produce more flow patterns, two things should happen: reduce the pipe diameter and upgrade the setup to inject larger amounts of air.

\section{ACKNOWLEDGEMENT}

This publication was made possible by the grant NPRP10-0101-170091 from Qatar National Research Fund (a member of the Qatar Foundation). Statements made herein are solely the responsibility of the authors.

\section{REFERENCES}

[1] http://www.whatispiping.com/what-is-fluid-flow. Retrieved October 13, 2016.

[2] http://uorepc-nitk.vlabs.ac.in/exp1/index.html. Retrieved October 13, 2016.

[3] An Overview of Heat Transfer Phenomena, Two-Phase Flow by M. M. Awad, ISBN 978-953-51-0827-6, (11). 252-254.

[4] Dukler H.M. Mekisso, Comparison of frictional pressure drop correlations for isothermal two-phase horizontal flow, Bachelor of Science in Engineering, Bahir Dar University, Bahir Dar, Ethiopia 2004, 2(2), 33-34.

[5] https://neutrium.net/fluid_flow/pressure-loss-in-pipe/. Retrieved November 2, 2016.

[6] Dziubinski, M., and R. P. Chhabra. "Predicting 2-Phase Pressure-Drop for The Flow of Gas-Non-Newtonian Liquid-Mixtures in Horizontal Pipes." International Journal of Engineering Fluid Mechanics 2.1 (1989): 63-78.

[7] Ruiz-Viera, M. J., Delgado, M. A., Franco, J. M., Sánchez, M. C., \& Gallegos, C. (2006). On the drag reduction for the twophase horizontal pipe flow of highly viscous non-Newtonian liquid/air mixtures: Case of lubricating grease. International Journal of Multiphase Flow, 32(2), 232-247.

[8] Two-phase-flow regimes, Halldor Palsson, University of Iceland, Accessed, October 19, 2016, www.researchgate.net.

[9] An Overview of Heat Transfer Phenomena Edited by Salim N. Kazi, ISBN 978-953-51-0827-6, 538 pages, Publisher: InTech, Chapters published October 31, 2012 under CC BY 3.0 license.

[10] Morshed, M., Amin, A., Rahman, M. A., \& Imtiaz, S. (2016, July). Experimental and computational analysis of pressure response in a multiphase flow loop. In M. Ali, M. A. S. Akanda, \& A. M. Morshed (Eds.), AIP Conference Proceedings (Vol. 1754, No. 1, p. 040010). AIP Publishing.

[11] Hubbard M.G. \& Dukler A.E., 1966, The characterization of flow regimes for horizontal two-phase flow, Proc. Heat Transfer and Fluid Mech. Institute. Stanford University Press -M. Saad \& J.A. Moller eds.

[12] Xu, J. Y., Gao, M. C., \& Zhang, J. (2014). Pressure Drop Models for Gas/Non-Newtonian Power-Law Fluids Flow in Horizontal Pipes. Chemical Engineering \& Technology, 37(4), 717-722.

[13] Majumder, S. K., Ghosh, S., Mitra, A. K., \& Kundu, G. (2010). Gas-Newtonian and gas-non-Newtonian slug flow in vertical pipe, Part I: Gas holdup characteristics. International Journal of Chemical Reactor Engineering, 8(1).

[14] https://www.electronicsdatasheets.com/manufacturers/newport-electronics/inc/parts/px409005dwu5v. Retrieved November 9, 2016.

[15] http://cuthbertsonlaird.co.uk/comark-indicators/415-comark-c9555-0-30-psi-manometer.html. Retrieved November 10, 2016. 